\title{
Information, Constraint and Meaning from Pre-Biotic World to a Possible Post-Human One. An Evolutionary Approach ${ }^{\dagger}$
}

\author{
Christophe Menant \\ Independent Scholar, Bordeaux, France; christophe.menant@hotmail.fr; Tel.: +33-06-1021-2106 \\ + Presented at the IS4SI 2017 Summit DIGITALISATION FOR A SUSTAINABLE SOCIETY, Gothenburg, \\ Sweden, 12-16 June 2017.
}

Published: 9 June 2017

\begin{abstract}
The presentation proposes to complement an existing development on meaning generation for animals, humans and artificial agents by looking at what could have existed at prebiotic times and what could be a post-human meaning generation. The core of the approach is based on an existing model for meaning generation: the Meaning Generator System (MGS). The MGS is part of an agent submitted to an internal constraint. The MGS generates a meaning when it receives information that has a connection with the constraint. The generated meaning is used by the agent to implement an action (physical, biological or mental) aimed at satisfying the constraint. The action can be in or out the agent. The purpose of the presentation is to widen the MGS approach in order to reach a coverage for information, constraint and meaning from a pre-biotic level to a possible post-human one. We present the MGS for animals, humans and artificial agents with the corresponding constraints. We then look at what could have been a constraint at a pre-biotic far from thermodynamic equilibrium level. At the other end of the spectrum we look at a possible posthuman status with an evolution of a 'limit anxiety' human constraint and also with AAs submitted to animal or human type constraints. Such approach links information science with physics, evolution, anthropology, semiotics and human mind. Continuations are proposed.
\end{abstract}

Keywords: information; meaning; constraint; representation; locality; evolution; pre-biotic; selfconsciousness; anxiety management; post-human; ethics

\section{Introduction}

Information and meaning are important parts of our everyday lives. They are also part of animal lives (the presence of a cat means 'danger' for a mouse). Such a perspective can be extended to artificial agents dealing with information and meaning as derived from the designers (an obstacle has a meaning for a robot programmed to avoid it). But in all cases an important point is that meaningful information (meanings) do not exist by themselves. Putting aside metaphysical hypothesis most of us would agree that meanings are generated by agents for given purposes, for specific constraints to be satisfied. As already presented, constraints for animals can be characterized by 'stay alive' and 'live group life', and the ones for artificial agents by 'as programmed by designer' [1]. Constraints for humans are more difficult to identify because the nature of human mind is not clearly understood and also because constraints can be unconscious. Taking into account these limited understandings, a system approach to meaning generation can be developed based on an agent having to satisfy an internal constraint related to its nature. This has been done with the Meaning Generator System (MGS) which is part of an agent submitted to an internal constraint. When the MGS receives information having a connection with the constraint it generates meaningful information that will be used by the agent to implement an action aimed a satisfying the constraint [2]. 
Some first results have been presented in an evolutionary background [1,3] and also for artificial agents [4]. These results have provided a first coverage of the life-human segment in terms of meaning generation for internal constraint satisfaction. We would like here to extend that segment on its both sides. First by looking at what could have been the status of a local constraint in a prebiotic universe. And then by considering a possible post-human status characterized by a better mastering of meaning generation related to anxiety management and by the availability of artificial agents submitted to animal or human intrinsic constraints. This would allow us to draw an evolutionary approach for information, constraint and meaning from a pre-biotic level to a possible post-human one. Such approach provides a continuous thread linking information science with physics, evolution, anthropology, semiotics and human mind. More is to be done on these subjects.

\title{
2. Method
}

The Meaning Generator System is represented in Figure 1. It is part of an agent submitted to an internal constraint. The generated meaning is information leading the agent to implement an action satisfying the constraint in a dynamic mode. The action can be internal or external to the agent, and it can be physical, biological or mental.

The MGS is usable for basic life: the meaning 'danger' is generated in a mouse submitted to a 'stay alive' constraint when she sees a cat. That generated meaning will trigger a hide or run away action to satisfy the constraint. Application to humans is more complex as the understanding of human constraints is limited by our lack of understanding of human mind. Regarding artificial agents, the meaning generation is based on derived constraints satisfaction. The MGS can also be used with semiotics when compared to a simplified version of the Peircean Interpreter [1].

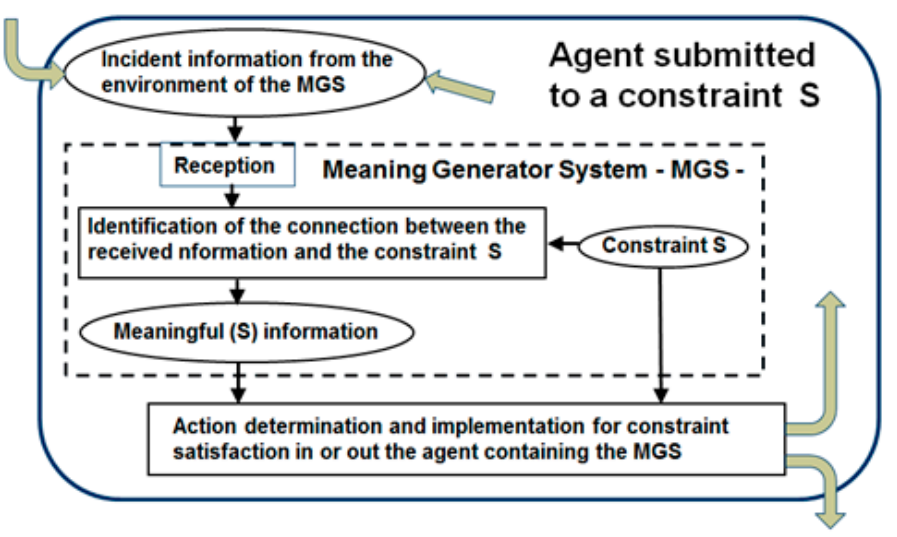

\author{
MGS: System approach \\ Animal constraints: \\ (Intrinsic) \\ - Stay alive (individual \& species) \\ - Live group life \\ Human constraints: \\ (Intrinsic) \\ - Limit anxiety \\ - Look for happiness \\ - Valorize ego,.... \\ Artificial Agent constraints: \\ (Derived) \\ - As programmed
}

Figure 1. The Meaning Generator System (MGS).

To reach some understanding of human constraints we use an evolutionary scenario for selfconsciousness that has introduced anxiety limitation as a generic human constraint [3]. The scenario is based on the evolution of meaningful representations. It presents an evolution toward an elementary form of self-consciousness (an 'ancestral self-consciousness') resulting from pre-human primates becoming able to represent their own entities as existing in the environment like conspecifics were represented. Such performance is assumed as having resulted from an evolution of inter-subjectivity into identification with conspecifics at pre-human times. The identification with conspecifics was also about identifications with suffering or endangered conspecifics that have produced within our ancestors a huge anxiety increase, an 'ancestral anxiety', having the same evolutionary origin that our ancestral self-consciousness. Anxiety limitation processes implemented to limit that anxiety increase have procured key evolutionary advantages and structured an 'evolutionary engine' favoring the evolution toward humans [3]. That evolutionary scenario links the nature of human consciousness to anxiety management. The evolutionary scenario is summarized in Figure 2. 


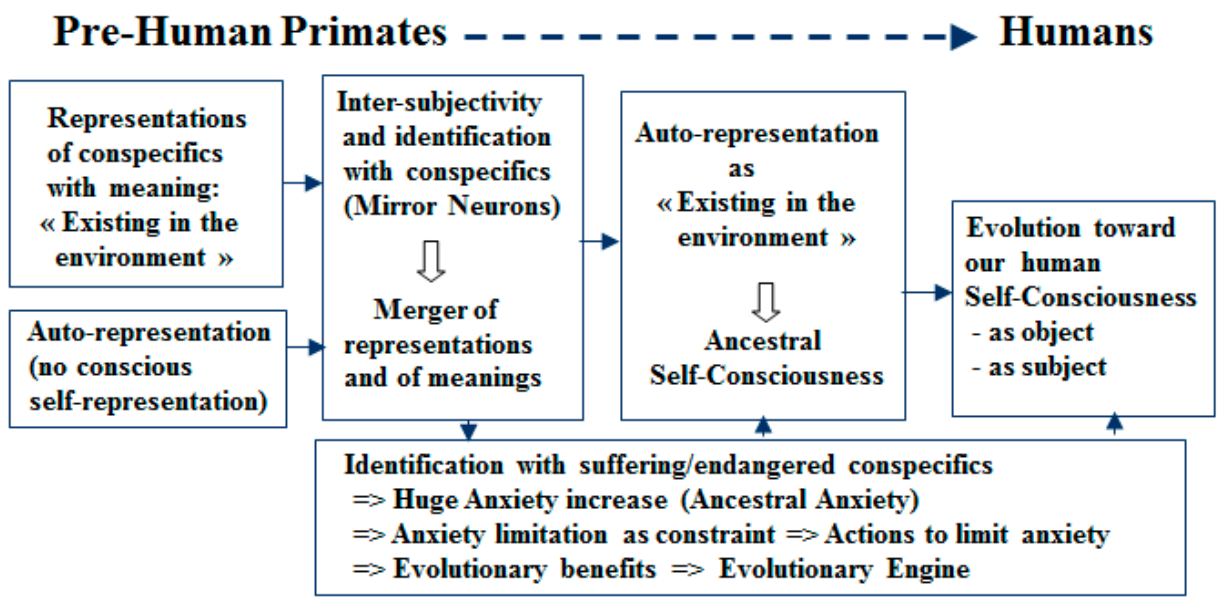

Figure 2. Meaning generation and self-consciousness (Short version of https://philpapers.org/rec/MENPFA-3).

Regarding a pre-biotic universe the presence of constraints has already been addressed by other authors in terms of emergence in a far from thermodynamic equilibrium [5]. This bring to propose an extension of the life-human segment to the pre-biotic world by using a local 'maintain status' constraint applied to a local far from thermodynamic equilibrium state [6].

On the other side of the life-human segment we propose a possible continuation of human evolution related to anxiety management and to artificial agents carrying animal or human constraints. The evolutionary scenario has shown that anxiety management is interwoven with the nature of self-consciousness [3]. We want to consider here a possible post-human status coming from a progressive mastering of the anxiety management processes leading to a less anxious human nature.

New artificial agents are also to come as part of a post-human status. Today artificial agents are human builds up with derived constraints programed by the human designer. Merging animal or human intrinsic constraints with the today derived ones present in AAs could introduce animal autonomy and human free will within these agents with associated ethical concerns [4]. Such an event is far from today scientific and philosophical achievements. We want to consider it as a possible post human status.

\section{Results and Discussion}

The above elements make available a background for an evolutionary continuity of meaningful information generation with local constraint satisfaction, starting at a pre-biotic level and going up to a possible post-human level.

The role of local/internal constraint satisfaction as a thread for the overall evolutionary process is summarized in Figure 3. It is worth noticing that the permanence in time of constraints could bring to introduce some characteristics of life at a pre-biotic level. 


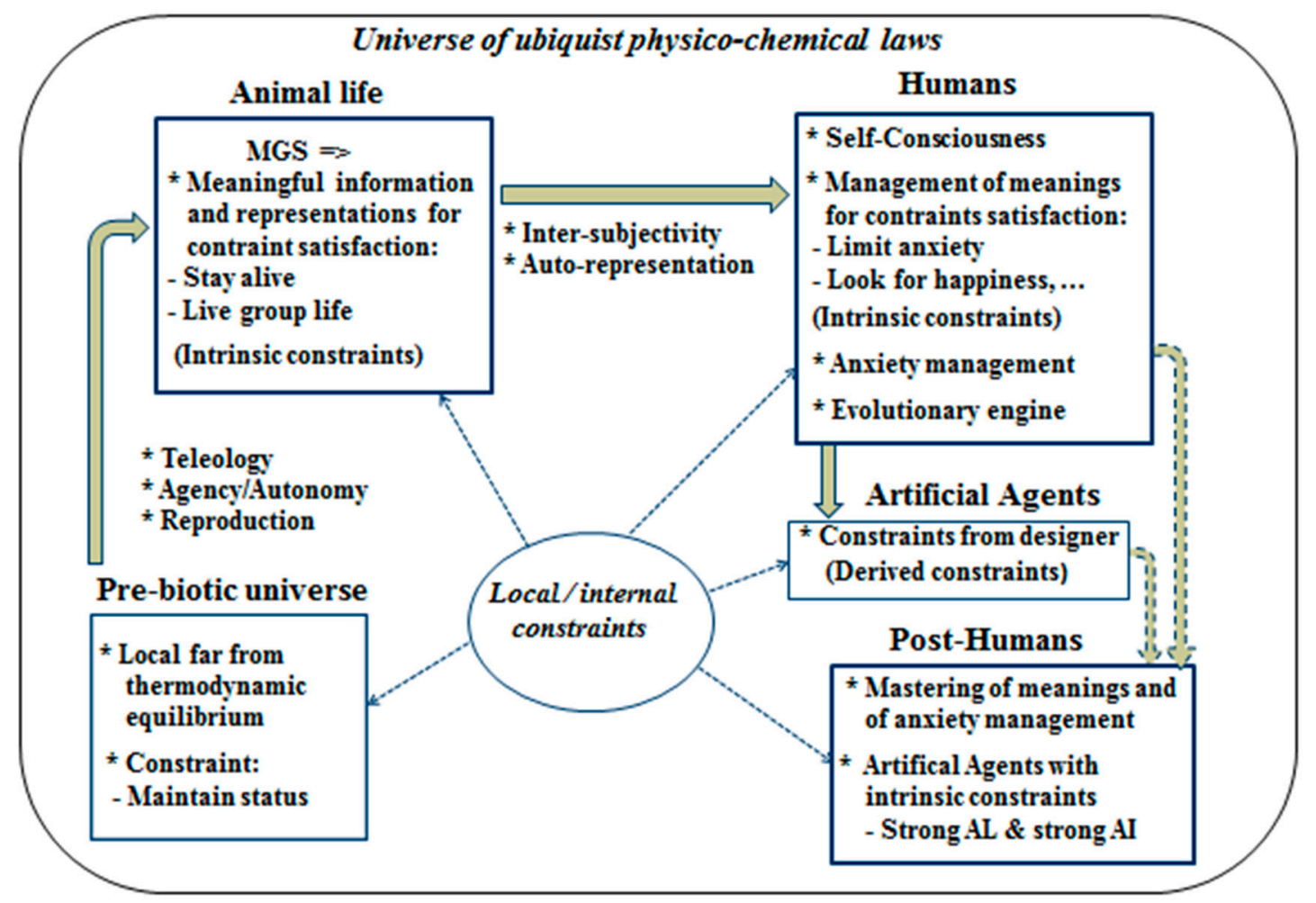

Figure 3. Evolutionary continuity in constraint satisfaction with meaning generation.

Several points of the presentation call for more developments, some related to ongoing activities in other areas:

- Concept of local constraint to be positioned in the evolution of an ubiquist a-biotic universe [7].

- Physics and information studies on the nature of a local far from thermodynamic equilibrium status maintaining itself [6].

- Pre-biotic sources of teleology, autonomy, agency and reproduction.

- Phylogenesis of human constraints in order to relate them to the evolution of human meaning generation, and consequently to human mind.

- Anthropology to study the shared evolutionary natures of self-consciousness and anxiety management as a key component of human mind.

- Psychology/psychiatry to consider phylogenesis of anxiety management as a source of human anxiety disorders and as a possible root of human evil [3].

- Philosophy of mind to deal with the evolution of ancestral self-consciousness into our today selfconsciousness as object and as subject.

- MGS structure to validate the concept of meaningful/meaningless information [1, 8].

- AI to consider its today limits as related to the unknown nature of life and address corresponding impact on artificial life [4].

- Possible merger of biological entities with artificial ones in terms of constraint satisfaction (merger of intrinsic and derived constraints). Address corresponding ethical concerns in terms of internal constraints satisfactions [4].

\section{Conclusions}

This presentation makes available an evolutionary thread of meaning generation for internal constraint satisfaction from pre-biotic entities up to possible post-humans ones. This comes in addition to already done work for animals and humans with the Meaning Generator System based on internal constraint satisfaction. The presentation goes with introducing some characteristics of life at a pre-biotic level and also highlights several points that need more developments in terms of 
information studies related to human evolution, to physics, to AI and to the evolution of meaning generation.

Conflicts of Interest: The authors declare no conflict of interest.

\section{References}

1. Menant, C. Computation on information, meaning and representations. Proposal for an evolutionary approach. In Information and Computation: Essays on Scientific and Philosophical Understanding of Foundations of Information and Computation; Dodig-Crnkovic, G., Burgin, M., Eds.; World Scientific: 2011; pp. 255-286. Available online: https://philpapers.org/rec/MENCOI (July 18th 2017).

2. Menant, C. Information and meaning. Entropy 2003, 5, 193-204. Available online: http://philpapers.org/rec/MENIAM-2 (July 18th 2017).

3. Menant, C. Proposal for an Evolutionary Nature of Self-Consciousness; 2014 paper. Available online: https://philpapers.org/rec/MENPFA-3 (July 18th 2017).

4. Menant, C. Turing Test, Chinese Room Argument, Symbol Grounding Problem. Meanings in Artificial Agents. In APA Newsletter fall 2013. Available online: https://philpapers.org/rec/MENTTC-2 (July 18th 2017).

5. Bickhard, M. Autonomy, Function, and Representation. Commun. Cognit. Artif. Intell. 2000, 17, 111-131. Available online: https://philpapers.org/rec/BICAFA (July 18th 2017).

6. Menant, C. Biosemiotics, Aboutness of Meaning and Bio-intentionality. Proposal for an Evolutionary Approach. Gatherings in Biosemiotics 2015, Copenhagen, Denmark, 30 June-4 July 2015. Available online: https://philpapers.org/rec/MENBAM-2 (July 18th 2017).

7. Pattee, H.H.; Kull, K. A biosemiotic conversation: Between physics and semiotics. Sign Syst. Stud. 2009, 37, 311-331. Available online: http://www.zbi.ee/ kalevi/11\%20Pattee-Kull.pdf (July 18th 2017).

8. Burgin, M. Is information some kind of data? In Proceedings of the Third Conference on the Foundation of Information Science (FIS 2005), Paris, France, 4-7 July 2005; pp. 1-31. Available online: http://www.mdpi.net/fis2005/F.08.paper.pdf (July 18th 2017).

(C) 2017 by the authors. Licensee MDPI, Basel, Switzerland. This article is an open access article distributed under the terms and conditions of the Creative Commons Attribution (CC BY) license (http://creativecommons.org/licenses/by/4.0/). 